\title{
Síndrome de Mazabraud: reporte de un caso
}

\author{
Rodrigo Re, " Maximiliano Negri,"* Jorge Flores" \\ "Servicio de Diagnóstico por Imágenes, Área Osteoarticular/Musculoesquelético - Intervencionismo, Sanatorio Allende, Córdoba, Argentina \\ ** Servicio de Traumatología, Sanatorio Allende, Córdoba, Argentina \\ ${ }^{\#}$ Servicio de Anatomía Patológica, Sanatorio Allende, Córdoba, Argentina
}

\section{RESUMEN}

El síndrome de Mazabraud es una rara asociación de lesiones del sistema musculoesquelético. Resulta de la combinación de uno o varios mixomas intramusculares y displasia fibrosa. Presentamos a un hombre de 46 años con lesiones óseas compatibles con displasia fibrosa asociada a mixoma intramuscular configurando un síndrome de Mazabraud y una revisión de la bibliografía. Palabras clave: Síndrome de Mazabraud; displasia fibrosa; mixoma intramuscular.

Nivel de Evidencia: IV

\section{Mazabraud Syndrome: A Case Report}

\section{ABSTRACT}

Mazabraud's Syndrome is a rare association of lesions to the musculoskeletal system. It results from the combination of one or more intramuscular myxomas and fibrous dysplasia. We present a case of a 46-year-old man with bone lesions compatible with fibrous dysplasia associated with intramuscular myxoma, configuring Mazabraud's Syndrome and a review of the literature.

Keywords: Mazabraud's syndrome; fibrous dysplasia; intramuscular myxoma.

Level of Evidence: IV

\section{INTRODUCCIÓN}

La displasia fibrosa es una anomalía del desarrollo esquelético de etiología desconocida; se caracteriza por la sustitución de la cavidad medular por tejido fibroso. Puede presentarse como una lesión única (monostótica) o múltiples lesiones (poliostótica). ${ }^{1}$ Produce fragilidad ósea con deformidad, dolor, fracturas patológicas e impotencia funcional. La displasia fibrosa, por lo general, se localiza en la pelvis y el fémur. En las radiografías, se visualiza como lesiones diafiso-metafisarias, líticas o densas, localizadas o difusas, que pueden expandir el hueso y generar un aspecto trabeculado, como de "vidrio esmerilado". ${ }^{2}$

La asociación entre displasia fibrosa y mixomas intramusculares se denomina síndrome de Mazabraud. El primer caso fue descrito por Henschen, en $1926^{3}$ y nombrado por Mazabraud, en 1957. ${ }^{4}$ Se han comunicado aproximadamente 100 casos hasta la fecha. ${ }^{1}$

El mixoma intramuscular es una lesión rara, con diagnóstico radiológico y clínico difícil. Su incidencia es de 1:1.000.000 personas. Es una neoplasia benigna de tejidos blandos de origen mesenquimatoso. ${ }^{5}$ Típicamente involucra músculos, muslos, glúteos, hombros y la parte superior del brazo. Desde el punto de vista clínico, los mixomas intramusculares se presentan como una masa indolora, ovoidea y de crecimiento lento. ${ }^{6-9}$

\section{CASO CLÍNICO}

Hombre de 46 años, sin antecedentes patológicos, que consulta a un médico clínico en otro centro, en octubre de 2019, por dolor lumbar mecánico localizado durante la actividad física, de varios meses de evolución. Se le solicita una resonancia magnética de columna y pelvis, en la que se observa una imagen dudosa en el fémur proximal, por lo que se decide extender el estudio al muslo y complementarlo con radiografías y tomografía computarizada de todo el miembro inferior derecho. 
En febrero de 2020, consulta a un traumatólogo de nuestro centro. En el examen físico, la movilidad de la cadera y la rodilla es completa, sin dolor en el miembro inferior derecho. Además, se palpa una tumoración en la zona anteroexterna proximal de la pierna derecha de aproximadamente $8 \mathrm{~cm}$, profunda, elástica, no dolorosa (Figura 1) que, según el paciente, tenía desde los 17 años de edad y su crecimiento era lento. Se solicitan nuevas radiografías y una resonancia magnética de cadera y pierna, y una ecografía de la masa de partes blandas en la pierna (Figura 2).

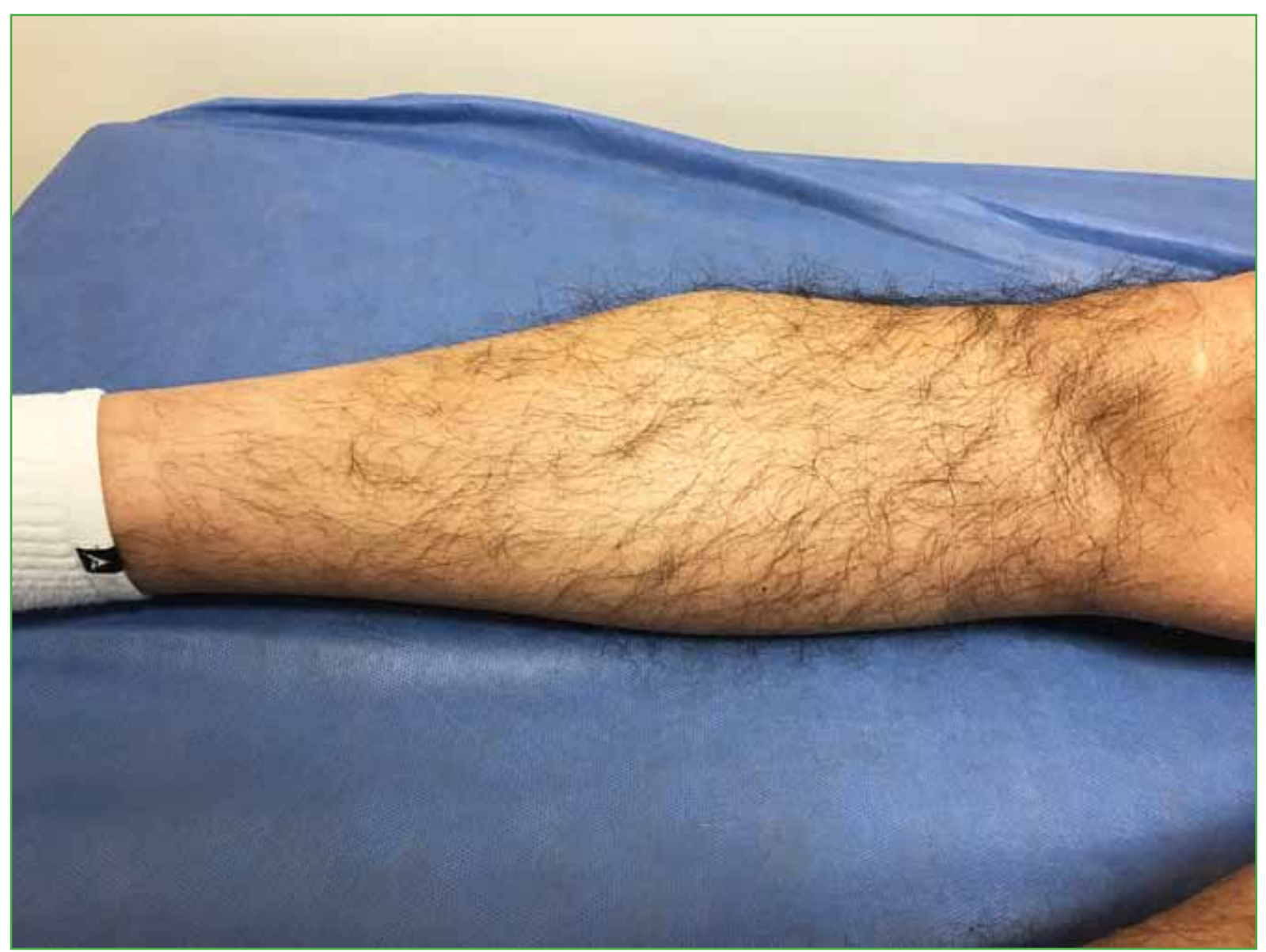

Figura 1. Imagen clínica de la lesión. 

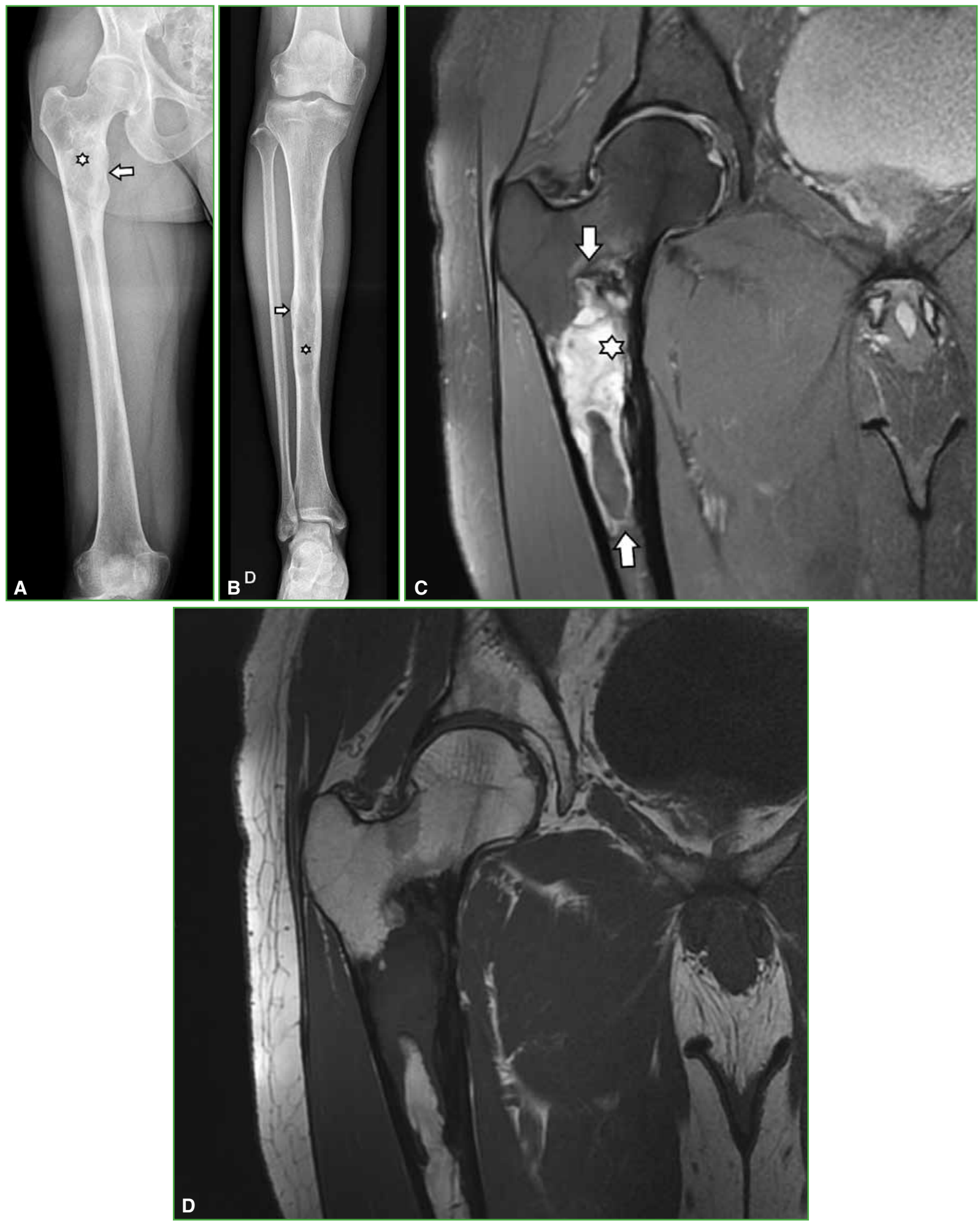

Figura 2. A. Radiografía de frente, de fémur derecho. Abombamiento óseo (asterisco) del cuello femoral adyacente al trocánter menor, adelgazamiento cortical (flecha), con pérdida del patrón trabecular. B. Radiografía de pierna derecha con tres lesiones de similares características a las descritas en el fémur, compromiso endóstico (flecha) y apariencia de "vidrio esmerilado" (asterisco). Resonancia magnética de cadera derecha. C. Corte coronal, secuencia STIR. Marcada hiperseñal, compromiso endóstico (asterisco), abombamiento óseo y límites definidos (flechas). D. Resonancia magnética de cadera derecha, corte coronal, secuencia T1 con marcada hiposeñal. 

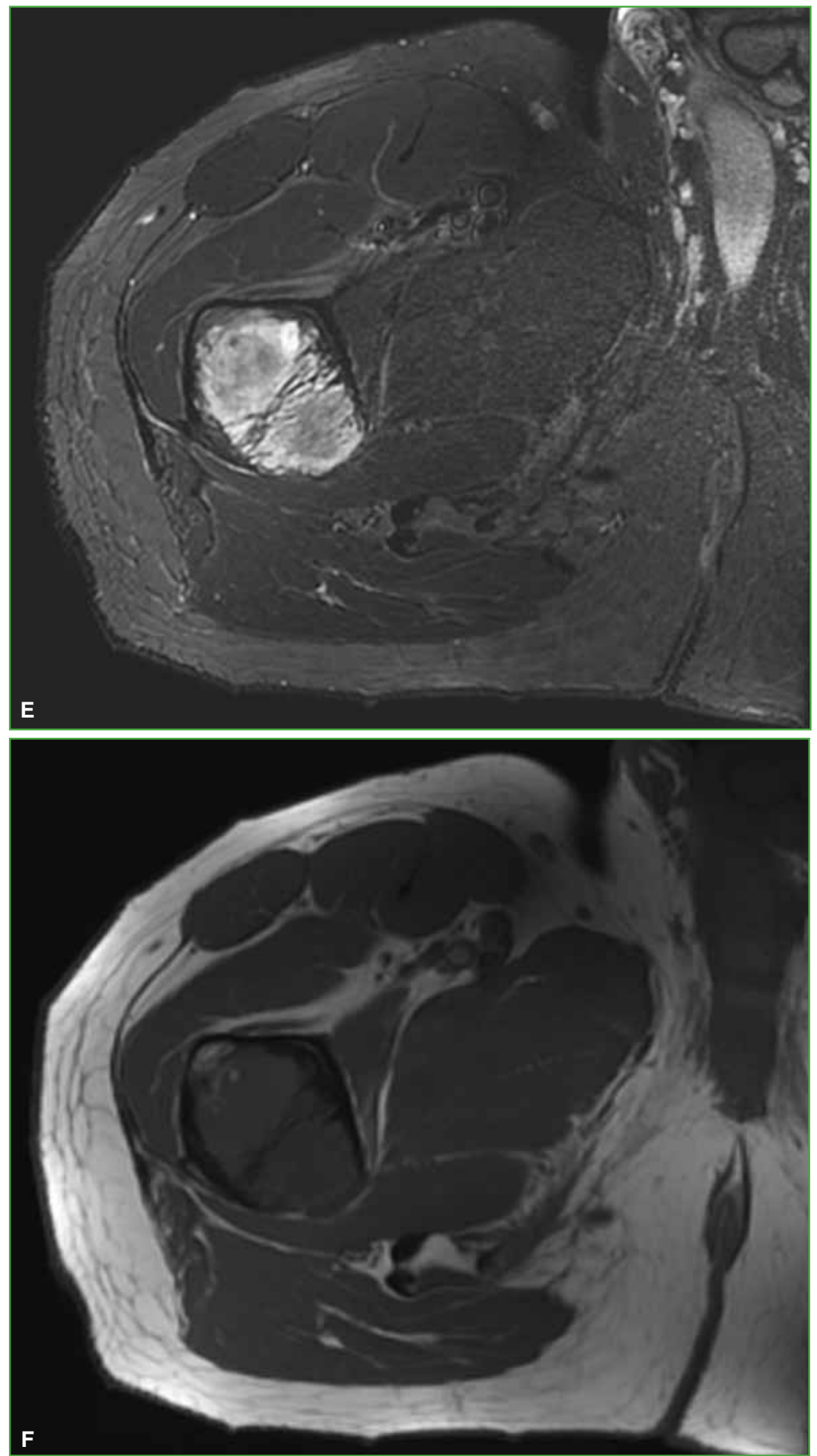

Figura 2. E. Resonancia magnética de cadera derecha, corte axial, secuencia STIR con hiperseñal. Abombamiento óseo y finos tabiques en el interior. F. Resonancia magnética de cadera derecha, corte axial, secuencia $\mathrm{T} 1$ con hiposeñal. 

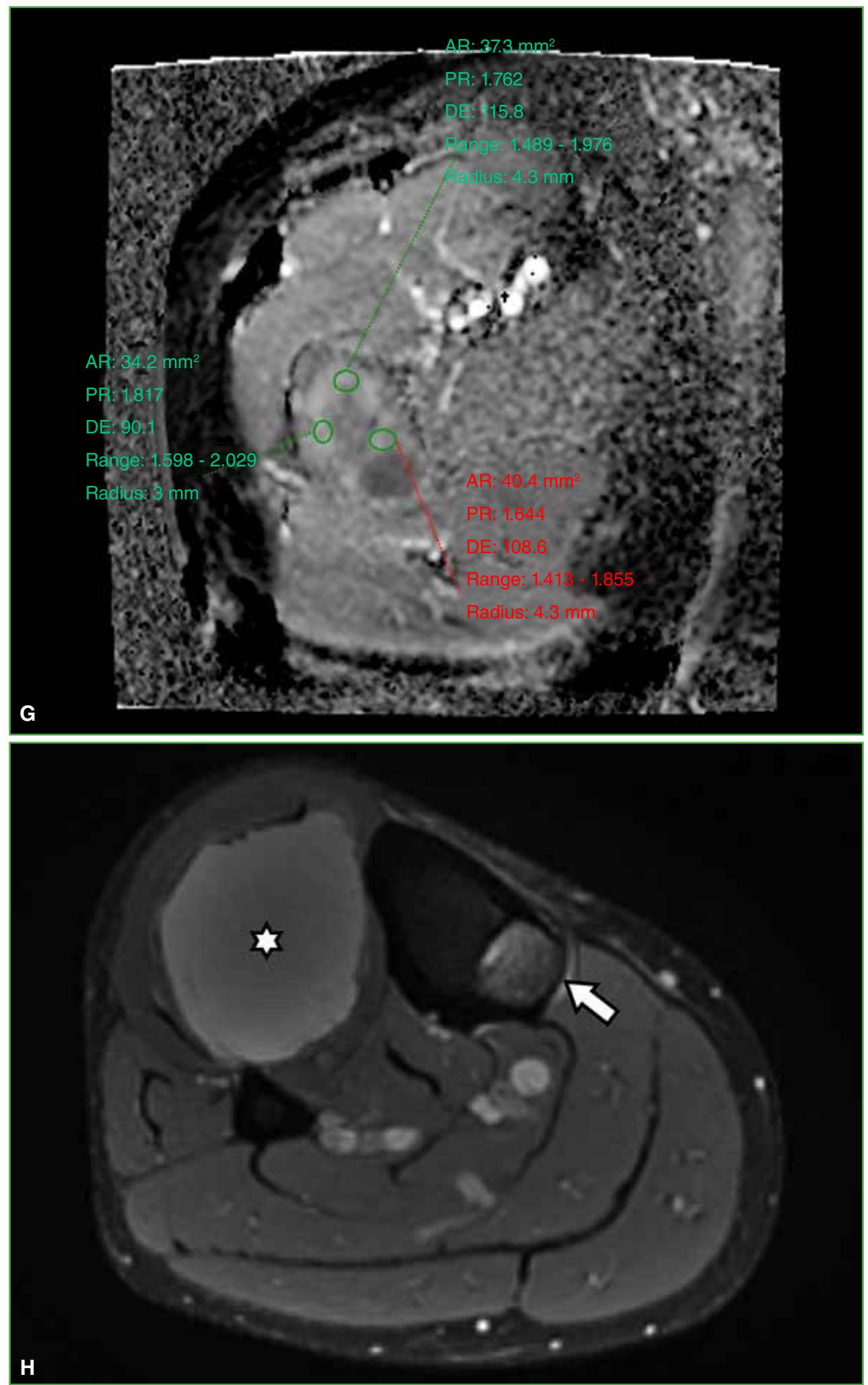

Figura 2. G. Mapa de coeficiente de difusión aparente con valor promedio de $1,7 \times 10^{-3} \mathrm{~mm}^{2} / \mathrm{s}$, marcando una difusión facilitada. H. Resonancia magnética de pierna derecha, corte axial, secuencia STIR. Lesión hiperintensa en la tibia proximal (flecha) y lesión marcadamente hiperintensa en partes blandas, en el plano muscular anteroexterno (asterisco). 

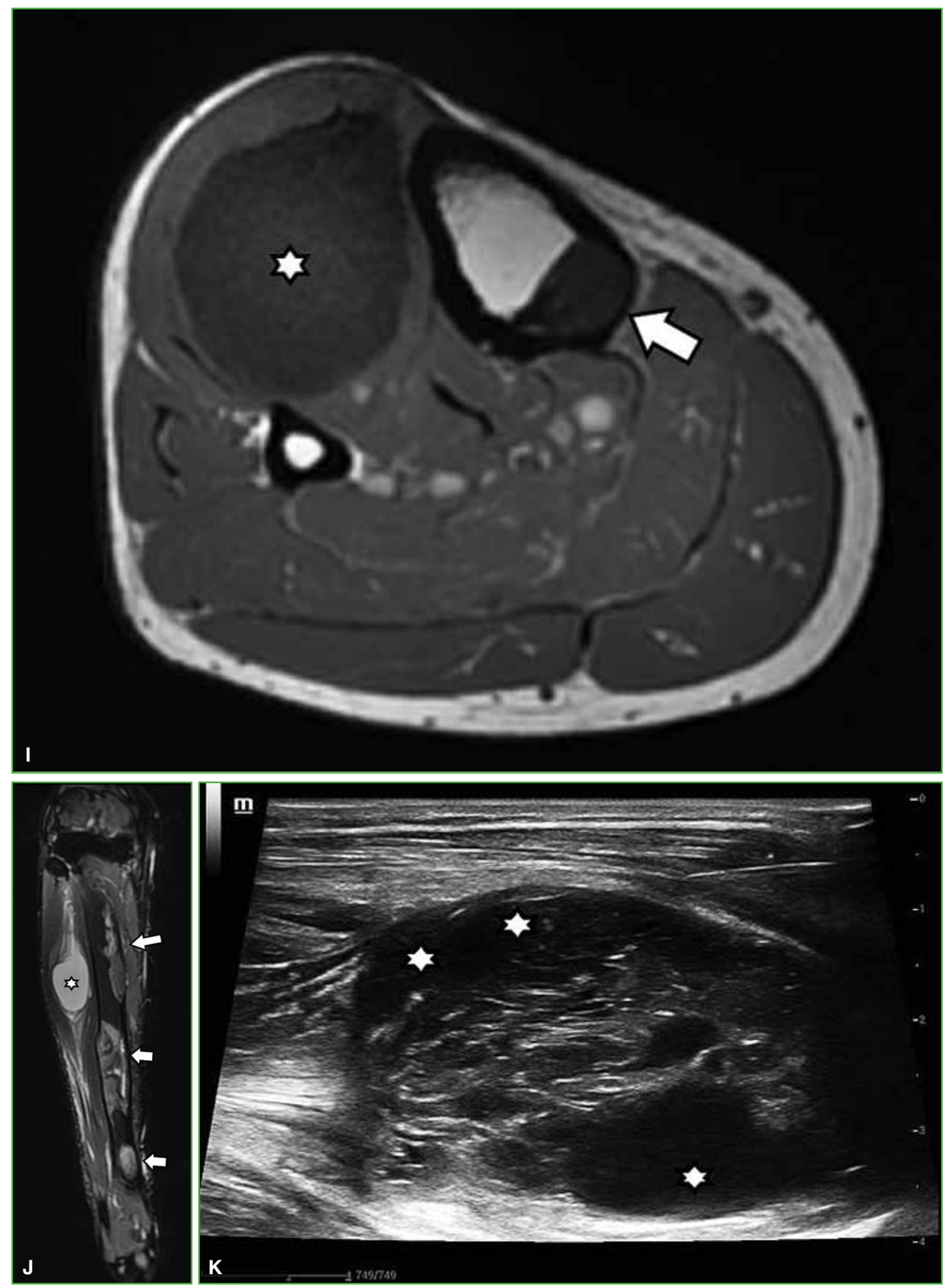

Figura 2. I. Resonancia magnética de pierna derecha, corte axial, secuencia T1. Lesión hipointensa en la tibia proximal (flecha) y lesión marcadamente hipointensa en partes blandas (asterisco). J. Resonancia magnética de pierna derecha, corte coronal, secuencia STIR. Múltiples lesiones hiperintensas óseas (flechas) y lesión hiperintensa en partes blandas (asterisco). K. Ecografía de la lesión en partes blandas. Morfología ovoidea, con bordes parcialmente definidos, heterogénea, con pequeñas áreas quísticas periféricas (asteriscos). 
Ante la sospecha de una asociación entre lesiones óseas, interpretadas como displasia fibrosa y lesión de partes blandas, se solicita una biopsia de la masa de partes blandas (Figura 3), con confirmación histológica de mixoma intramuscular (Figura 4), que configura un síndrome de Mazabraud.

El paciente continuará en observación con estudios radiológicos y de evolución clínica.

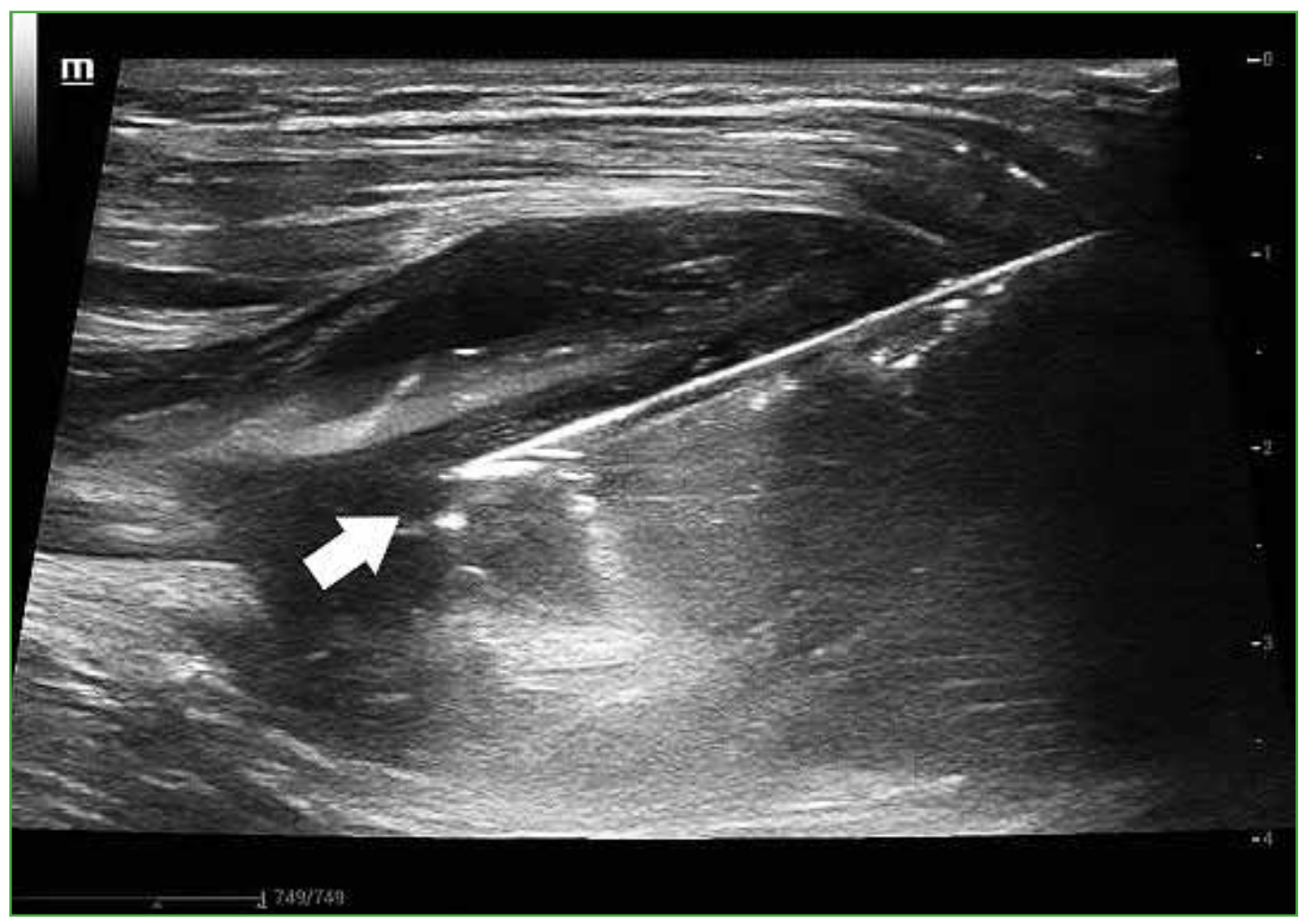

Figura 3. Biopsia de la lesión de partes blandas guiada por ecografía con aguja de corte (flecha). 

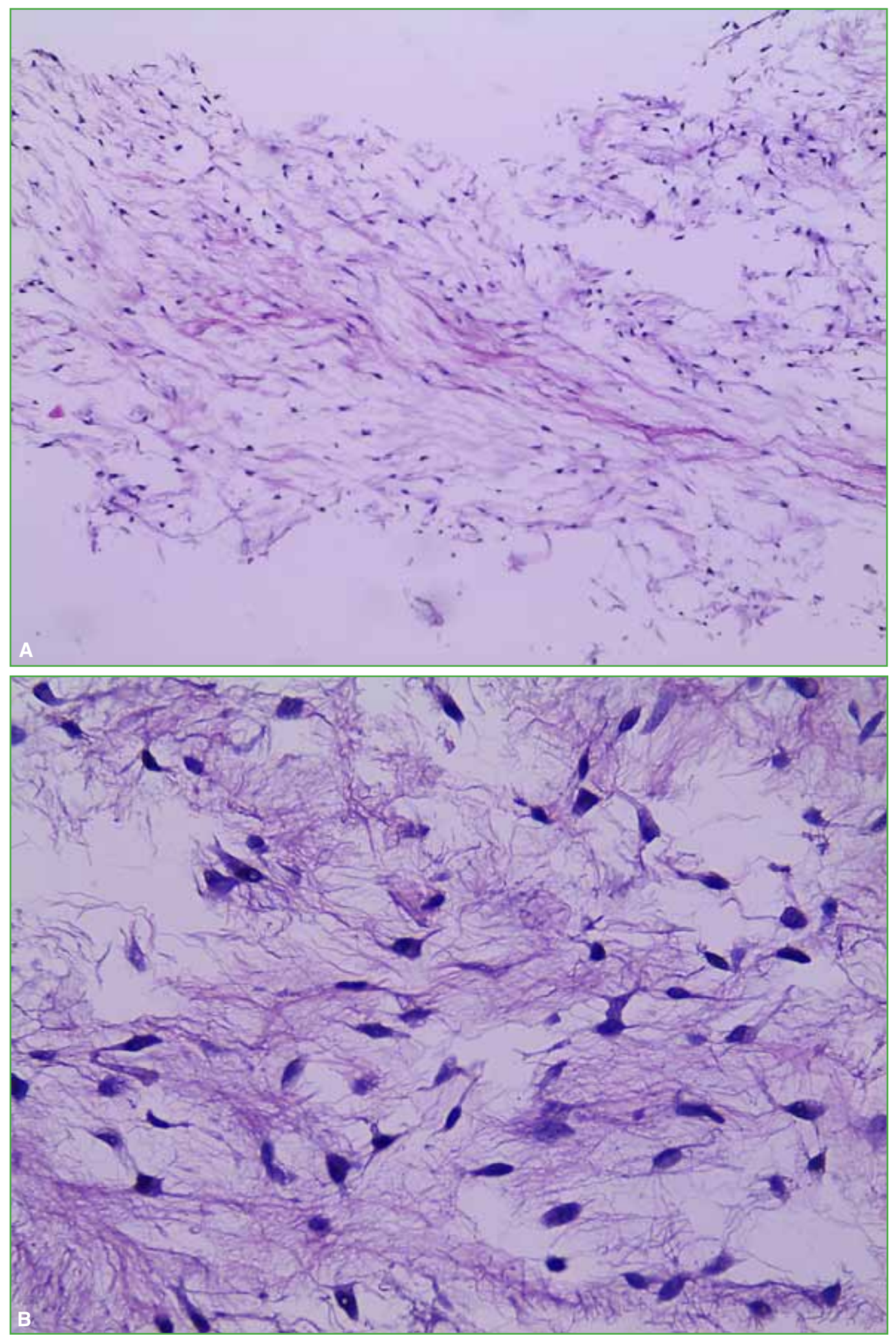

Figura 4. Anatomía patológica con tinción de hematoxilina-eosina. Mediano (A) y gran aumento (B).

Se visualiza un tumor mesenquimatoso con estroma mixoide fusocelular. No hay atipia, mitosis, necrosis ni otras alteraciones de importancia. 


\section{DISCUSIÓN}

La displasia fibrosa es una lesión benigna infrecuente causada por una mutación genética en el gen GNAS, localizado en el cromosoma 20q13.2-q13.3. ${ }^{10}$ Se caracteriza por la presencia de áreas no osificadas en el hueso. Puede ser monostótica o poliostótica, y es más común en las mujeres $(68 \%) \cdot{ }^{1,2,10}$ En general, se diagnostica entre la primera y la tercera década de la vida. Los sitios más afectados son el fémur proximal, la tibia, el cráneo, las costillas y, en menor medida, el húmero, el antebrazo y la pelvis. Con frecuencia, hay varias afecciones en un mismo hueso.

La variante monostótica se presenta sin síntomas, como un hallazgo incidental. La variante poliostótica puede cursar con dolores intermitentes, fracturas por fatiga, masas por expansión en huesos palpables o fracturas patológicas. La asociación de displasia fibrosa poliostótica con mixoma intramuscular configura el síndrome de Mazabraud. ${ }^{1,2,9,10}$

Los estudios por imágenes de la displasia fibrosa son importantes para el diagnóstico apropiado y el seguimiento, a fin de detectar y tratar posibles complicaciones.

En las radiografías, se visualiza como lesiones intramedulares diafisarias, radiolúcidas, con apariencia de "vidrio esmerilado" y pérdida del patrón trabecular normal, compromiso endóstico y adelgazamiento de la cortical con áreas respetadas. Los márgenes son definidos. No se observa una reacción perióstica. Las imágenes de la tomografía computarizada confirman la presencia de lesiones homogéneas con apariencia de "vidrio esmerilado". Puede haber áreas quísticas y calcificaciones. En la resonancia magnética, se observan lesiones con baja señal en las secuencias T1 y alta señal en las secuencias T2. Puede haber un halo hipointenso a su alrededor en las secuencias T1 y T2. Como es una lesión benigna, tiene difusión facilitada con valores de coeficiente de difusión aparente por encima de $1,1 \times 10^{-3} \mathrm{~mm}^{2} / \mathrm{s}^{1,2,10}$

Las radiografías son normales, aunque los mixomas intramusculares se pueden manifestar con calcificaciones no específicas en partes blandas. En la ecografía, los mixomas intramusculares se visualizan como masas ovoideas, poco definidas e hipoecoicas, con posibles áreas quísticas. En la tomografía computarizada, aparecen como lesiones hipointensas con pared que las separa del tejido muscular. En la resonancia magnética, se visualizan como lesiones hiperintensas en las secuencias T2, gradiente de eco o STIR. Las secuencias T1 muestran baja señal.,6-9

\section{CONCLUSIONES}

A pesar de que la asociación entre displasia fibrosa y mixomas intramusculares es rara, probablemente esté subestimada debido a diagnósticos erróneos. La displasia fibrosa, por lo general, se diagnostica antes que los mixomas intramusculares o en el mismo momento, como hallazgos en radiografías de rutina o resonancia magnética.

Los síntomas son raros, se pueden manifestar con fracturas patológicas o masas palpables.

El tratamiento de los mixomas intramusculares es la escisión quirúrgica. La displasia fibrosa se controla con estudios por imágenes. La posibilidad de transformación maligna es baja.

Conflicto de intereses: Los autores no declaran conflictos de intereses.

\section{BIBLIOGRAFÍA}

1. Vescini F, Falchetti A, Tonelli V, Carpentieri M, Cipri C, Cosso R, et al. Mazabraud's syndrome: a case report and up-to-date literature review. Endocr Metab Immune Disord Drug Targets 2019;19(6):885-93. https://doi.org/10.2174/1871530319666181226103700

2. Case DB, Chapman CN Jr, Freeman JK, Polga JP. Atypical presentation of polyostotic fibrous dysplasia with myxoma (Mazabraud syndrome). RadioGraphics 2010;30:827-32. https://doi.org/10.1148/rg.303095155

3. Henschen F. Fall von ostitis Fibrosa mit multiplen Tumoren in derumgebenden Muskulatur. Verh Dtsch Ges Pathol 1926;21:93-7. 
4. Mazabraud A, Girard J. [A peculiar case of fibrous dysplasia with osseous and tendinous localizations]. Rev Rhum Mal Osteoartic 1957;24(9-10):652-9. PMID: 13518962

5. Stout A. Myxoma: the tumor of primitive mesenchyme. Ann Surg 1948;127(4):706-19. PMID: 18917127

6. Nielsen GP, O'Connell JX, Rosenberg AE. Intramuscular myxoma: a clinicopathologic study of 51 cases with emphasis on hypercellular and hypervascular variants. Am J Surg Pathol 1998;22(10):1222-7. https://doi.org/10.1097/00000478-199810000-00007

7. Murphey MD, McRae GA, Fanburg-Smith JC, Levine AM, Aboulafia AJ. Imaging of soft-tissue myxoma with emphasis on CT and MR and comparison of radiologic and pathologic findings. Radiology 2002;225(1):215-24. https://doi.org/10.1148/radiol.2251011627

8. Nishio J, Naito M. FDG PET/CT and MR imaging of intramuscular myxoma in the gluteus maximus. World J Surg Oncol 2012;10(1):132. https://doi.org/10.1186/1477-7819-10-132

9. Baltu Y, Arikan ŞM, Dölen UC, Uzun H, Alkan Bİ, Aydın O. Intramuscular myxoma: clinical and surgical observation notes on eleven cases. Int Orthop 2017;41(4):837-43. https://doi.org/10.1007/s00264-016-3396-8

10. Zoccali C, Teori G, Prencipe U, Erba F. Mazabraud's syndrome: A new case and review of the literature. Int Orthop 2009;33(3):605-10. https://doi.org/10.1007/s00264-007-0483-X 